Check for updates

Cite this: RSC Adv., 2019, 9, 2431

Received 3rd December 2018

Accepted 6th January 2019

DOI: $10.1039 / c 8 r a 09948 k$

rsc.li/rsc-advances

\section{Novel ionic surface imprinting technology: design and application for selectively recognizing heavy metal ions $\dagger$}

\author{
Fu-Qiang An, (D) *a Hu-Fei Li, ${ }^{a}$ Xu-Dong Guo, ${ }^{b}$ Bao-Jiao Gao, ${ }^{a}$ Tuo-Ping Hu (D) ${ }^{a}$ \\ and Jian-Feng $\mathrm{GaO}^{\mathrm{a}}$
}

Traditional bulk polymerization imprinted technology and existing surface imprinted technology have some congenital defects. Therefore, it is necessary to design more efficient surface imprinted technology. In this paper, novel surface imprinting technology with higher imprinting efficiency is well designed. It fully realizes the synchronization of polymer crosslinking and template imprinting. Then the surface imprinted polymers (SIPs) are synthesized using metal ions as a template. The physicochemical characteristics of the SIPs are characterized by scanning electron microscopy (SEM), Brunauer-Emmett-Teller (BET) studies, Fourier transform infrared spectroscopy (FTIR) and elemental analysis. The adsorption performances and recognition selectivity of the SIPs towards the template are investigated by a batch method. The experimental results show that the SIPs possess excellent adsorption ability and selectivity towards the template. The selectivity coefficients of the SIPs prepared in this study are higher than those of IIPS prepared by other imprinting methods. The adsorption process could be well described by the Lagergren-first-order model and Langmuir monolayer chemical adsorption. The SIPs have good chemical stability and reusability. Consecutive adsorption-desorption experiments show that the exhausted SIPs could be effectively regenerated, and the regenerated SIPs could be reused without a significant reduction in adsorption capacity or selectivity coefficient.

\section{Introduction}

Molecule/ion imprinting technology is well known as an efficient approach to synthesize polymeric materials with tailor-made imprinted cavities. ${ }^{1,2}$ Like the relationship between key and lock, these imprinted cavities (lock) allow only designated molecules/ions (key) to enter and interact with them. So, the molecular/ionic imprinted polymers (MIPs/IIPs) possess high specificity and selectivity towards template molecules/ions, and they can selectively recognize templates at the molecular level. Due to the merits of high recognition selectivity, good mechanical and chemical stability, and preparation simplicity, MIPs/IIPs have been utilized in multiple applications, such as solid-phase extraction, in chromatographic separation, as sensors, in drug release and as catalysts, $e^{3 c^{3-6}}$.

The traditional synthetic method of MIPs/IIPs is a bulk polymerization method, and the synthesis processes are briefly

${ }^{a}$ Chemical Department, North University of China, Taiyuan 030051, People's Republic of China. E-mail: anfuqiang@nuc.edu.cn

${ }^{b}$ Codan-Lingyun Automotive Rubber Hose Co., Ltd, Zhuozhou 072750, People's Republic of China

$\dagger$ Electronic supplementary information (ESI) available. See DOI: $10.1039 / \mathrm{c} 8 \mathrm{ra} 09948 \mathrm{k}$ described below. ${ }^{7,8}$ Firstly, the functional monomers are selfassembled with templates by way of non-covalent or covalent interactions. Then the polymerization reaction of monomertemplate complexes and crosslinkers is carried out by adding an initiator. After the polymerization is complete, the template is eluted from the polymeric particles, and the imprinted cavities which are complementary to the template in size, shape and spatial arrangement of functional groups are generated in the polymer network. Finally, the obtained monoliths are ground and sieved to the required particle dimensions.

The MIPs/IIPs prepared by using a bulk polymerization method suffer some drawbacks, such as difficult template removal, slow mass transfer rate, and poor site accessibility for target species. These fatal drawbacks all result from the thick polymer layer and deeply embedded recognition sites. In order to overcome these drawbacks effectively, the recognition sites must be placed onto or near the substrate surface. Therefore, surface imprinting technology (SIT) has been developing rapidly under the unremitting efforts of researchers. ${ }^{9-13}$ Surface imprinted polymers (SIPs) have a thin polymer layer, which facilitates the removal and diffusion of templates.

SIT can be divided into two kinds of methods: one based on emulsion and precipitation polymerization ${ }^{14-17}$ and the other based on surface grafting and crosslinking..$^{18,19}$ The latter method is more efficient and is usually defined as a "pre- 
grafting polymerization and post-crosslinking/imprinting" method $^{19,20}$ or "grafting polymerization synchronized with crosslinking/imprinting" method..$^{21,22}$ The processes are briefly described as follows and shown in Fig. 1S and $2 \mathrm{~S}, \dagger$ respectively.

The "pre-grafting polymerization and post-crosslinking/ imprinting" method: (1) functional polymers with a strong interaction towards the templates are pre-grafted (in the manner of "grafting from" or "grafting to") onto the carrier surface, and the grafted particles form a thin grafted polymer layer; (2) the adsorption of the grafted particles towards templates reaches saturation by means of the interaction between the grafted functional polymers and the templates; (3) post-imprinting of templates is carried out by crosslinking the grafted polymers using a special crosslinking agent. After eluting the templates, a large number of imprinted caves complementary to the templates are formed and distributed within the thin crosslinked polymer layer, and the imprinted materials are synthesized.

The "grafting polymerization synchronized with crosslinking/imprinting" method: (1) a combination of the functional monomer and templates is formed on the basis of the interaction between them; (2) surface-initiated graftpolymerization is carried out synchronously with the crosslinking of the functional monomer and the crosslinking agent that is, the imprinting process of the templates under the action of an initiator. The thin crosslinked polymer layer in which lots of templates are embedded is grafted onto the carrier surface. After eluting the templates, a large number of imprinted caves are formed, and the imprinted materials are obtained.

Lots of surface imprinted materials are synthesized by using these two surface imprinting methods. However, some shortcomings have been found with their further application and research. For the "pre-grafting polymerization and post-crosslinking/imprinting" method, the grafted polymers must have two kinds of functional groups, one used to interact with the templates and another reacting with the crosslinking agent. This has higher requirements for grafted functional polymers. Furthermore, the crosslinking of the grafted polymers after adsorbing the templates is crucial. But the heterogeneity of the polymer reaction results in low crosslinking/imprinting efficiency, such as some templates not being imprinted. For the "grafting polymerization synchronized with crosslinking/ imprinting" method, some templates could affect the grafting polymerization due to their chain termination characteristics, and this decreases the grafting efficiency. More disadvantageously, a large number of traditional imprinted polymers have been formed through bulk polymerization. This greatly affects the synthesis of surface imprinted polymers.

The purpose of this paper is to design a simple and effective surface imprinting method. In this paper, a novel surface imprinting technology is originally designed and used to synthesize the surface imprinted material. This novel surface imprinting technology combines the advantages of the above two surface imprinting methods, and avoids the heterogeneity of the polymer reaction and the formation of bulk copolymer in the system. It fully realizes the synchronization of polymer crosslinking and template imprinting, and the imprinting efficiency is greatly improved. This novel surface imprinting technology further enriches surface imprinting technology and provides a new idea for the synthesis of surface imprinted materials. The synthesis process is described briefly in Fig. 1 . Firstly, polymers with reactive groups are grafted (in the manner of "grafting from" or "grafting to") onto the carrier surface. Meanwhile the templates are combined with ligands of the crosslinking agent. Then, the combination is mixed with the grafted carrier, and the crosslinking reaction of crosslinking agents combining with templates towards grafted polymers is carried out. That is, the crosslinking of grafted polymers is carried out synchronously with the imprinting of the templates. After eluting the templates, a large number of imprinted caves are formed, and the surface imprinted materials are obtained.

In order to demonstrate the progressiveness of the designed surface imprinting technology, a series of excellent surface imprinting materials are synthesized using heavy metal ions as the template, and the adsorption performances and recognition selectivity are investigated.

\section{Experimental}

\subsection{Materials and instruments}

D301 styrene macroporous resin was provided freely by Wandong Chemical Co., Ltd. (Anhui, China). Glycidyl methacrylate (GMA) was purchased from Ruijinte Chemical Ltd. (Tianjin, China) and purified by distillation under vacuum before use. Ethylenediamine was purchased from Beijing Chemical Plant (Beijing, China, AR grade). $\mathrm{Cd}\left(\mathrm{NO}_{3}\right)_{2}, \mathrm{~Pb}\left(\mathrm{NO}_{3}\right)_{2}, \mathrm{Cu}\left(\mathrm{NO}_{3}\right)_{2}$, $\mathrm{Zn}\left(\mathrm{NO}_{3}\right)_{2}$, and other reagents were purchased from Sinopharm Chemical Reagent Beijing Co., Ltd.

The instruments used in this study were as follows: AutosorbiQ surface area analyzer (Quantachrome Company, America), S4800 scanning electron microscope (Hitachi Company, Japan), Prodigy ICP-AES (Teledyne Leeman Lab, America), FTIR-8400S infrared spectrometer (Shimadzu, Japan), Vario EL elemental analyzer (Elementar, Germany), PHS-3 acidimeter (Shanghai INESA Scientific Instrument Co., Ltd., China), and SHZ-C waterbathing constant temperature shaker (Shanghai Boxun Medical Biological Instrument Co., Ltd., China).

\subsection{Preparation and characterization of surface imprinted materials}

Firstly, the GMA was grafted onto the surface of D301 resin by polymerization according to ref. 23 and the synthetic material was labelled D301-g-PGMA. The grafting degree (the quality of PGMA per gram of D301-g-PGMA) of PGMA is $0.42 \pm 0.05 \mathrm{~g} \mathrm{~g}^{-1}$. $1 \mathrm{~g}$ of D301-g-PGMA was soaked in $10 \mathrm{ml}$ of dimethyl formamide at room temperature for $12 \mathrm{~h}$. Meanwhile $1 \mathrm{~g}$ of $\mathrm{Cd}\left(\mathrm{NO}_{3}\right)_{2}$ and $0.6 \mathrm{ml}$ of ethylenediamine were added into $50 \mathrm{ml}$ of distilled water, and this content was stirred continuously at $323 \mathrm{~K}$ for $1 \mathrm{~h}$. Then, the mixture of $\mathrm{Cd}\left(\mathrm{NO}_{3}\right)_{2}$ and ethylenediamine was added dropwise into dimethyl formamide soaked D301-g-PGMA, and the ring-opening reaction of ethylenediamine and PGMA was carried out at $353 \mathrm{~K}$ for $8 \mathrm{~h}$. Subsequently, the resultant particles were washed by $1 \mathrm{~mol} \mathrm{~L}^{-1}$ of nitric acid to remove Cd(II) until no 


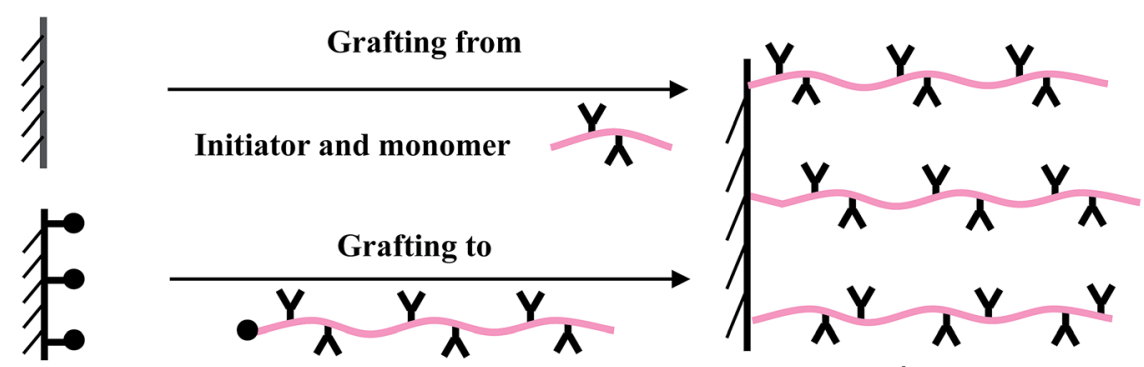

Polymers with reactive groups
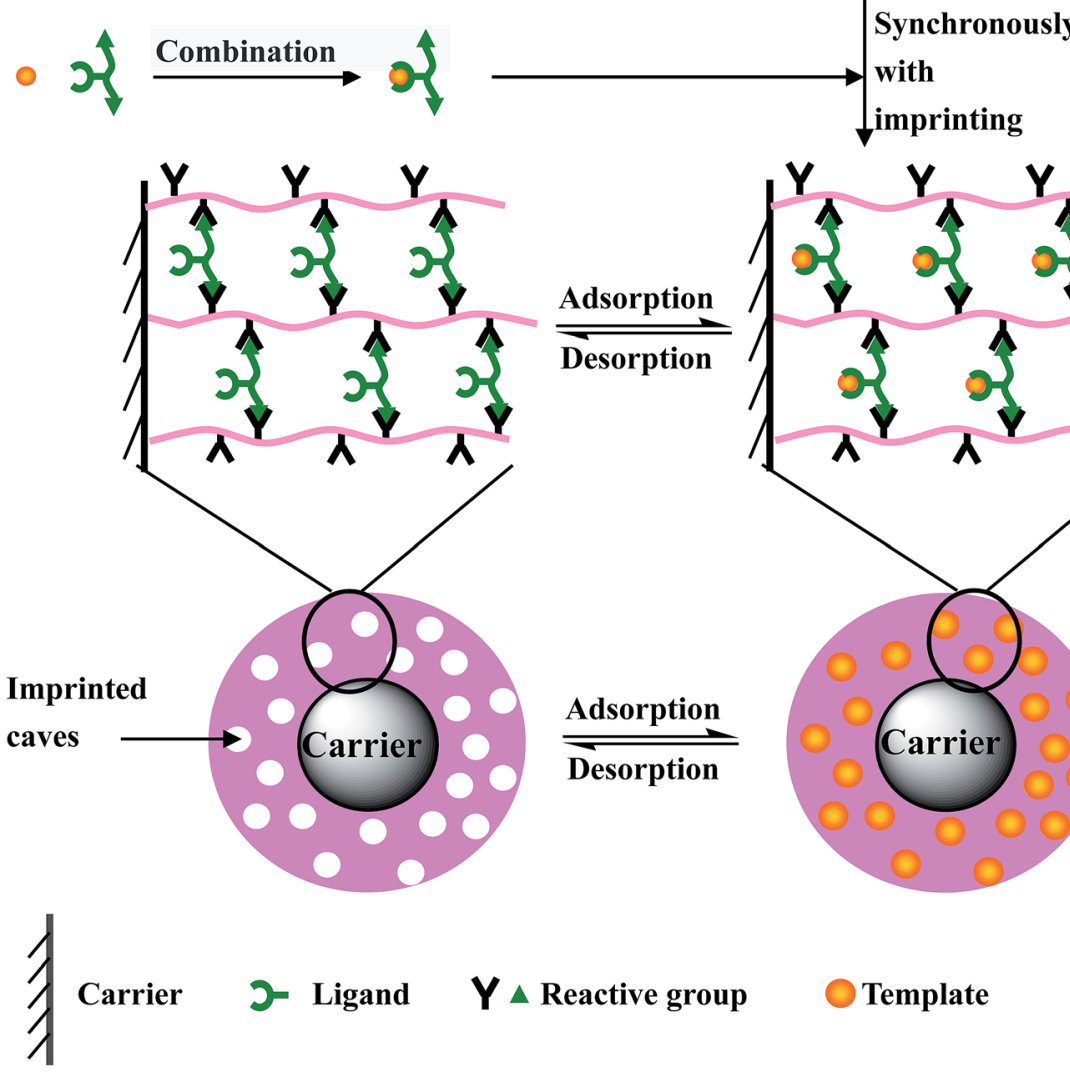

Fig. 1 The schematic diagram of the surface imprinted method designed in this study.

Cd(II) was tested by ICP in the eluent, and then washed with distilled water to neutrality. Finally, the Cd(II) surface imprinted materials were obtained and labeled Cd-SIP. The preparation process is expressed in Fig. 2. In the same way, the surface imprinted materials of different metal ions ( $\mathrm{Pb}(\mathrm{II}), \mathrm{Cu}$ (II), $\mathrm{Zn}$ (II)) were prepared by the same procedure. As a comparison, nonimprinted polymer (NIP) was also prepared in parallel but without the addition of metal ions in the preparation procedure.

\subsection{Adsorption experiments}

Adsorption experiments were performed using about $0.05 \mathrm{~g}$ of as-prepared SIPs and $200 \mathrm{ml}$ of metal ion (Cd(II), $\mathrm{Pb}$ (II), $\mathrm{Cu}(\mathrm{II}), \mathrm{Zn}(\mathrm{II}))$ solution by the batch method. The concentration of metal ions was tested using ICP-AES. The influences of contact time (0-40 $\mathrm{min})$, initial concentration $(0-4 \mathrm{mmol}$ $\left.\mathrm{L}^{-1}\right)$, and $\mathrm{pH}(0-6)$ on the adsorption capacity were investigated systematically. Each experiment was replicated 3 times in order to verify consistent results and the results were averaged. The adsorption capacity was calculated according to eqn (1).

$$
Q=\frac{V\left(C_{0}-C_{t}\right)}{m}
$$

where $Q\left(\mathrm{mmol} \mathrm{g}^{-1}\right)$ is the adsorption capacity, $V(\mathrm{~L})$ is the volume of the solution, $C_{0}$ and $C_{t}$ are the concentrations of metal ions at the start and at time $t$ in the solution $\left(\mathrm{mmol} \mathrm{L}^{-1}\right)$, and $m(\mathrm{~g})$ is the adsorbent dosage.

\subsection{Selectivity experiment}

In order to show the specific selectivity of as-prepared SIPs, Cd-SIP, $\mathrm{Pb}-\mathrm{SIP}, \mathrm{Cu}$ (II)-SIP, and Zn(II)-SIP were synthesized. The competitive adsorptions towards the template with respect to competitive ions were carried out by the batch method using $0.05 \mathrm{~g}$ of SIPs and 


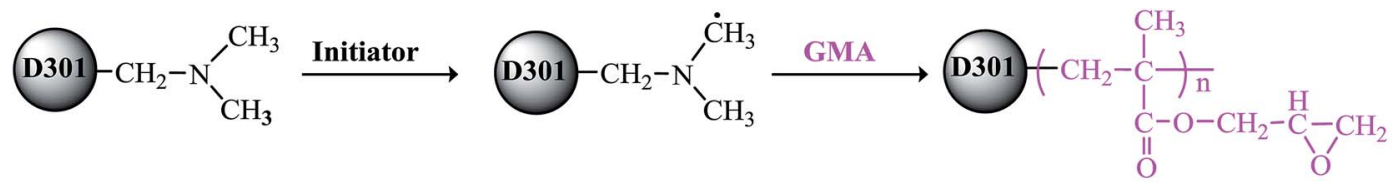

D301-g-PGMA
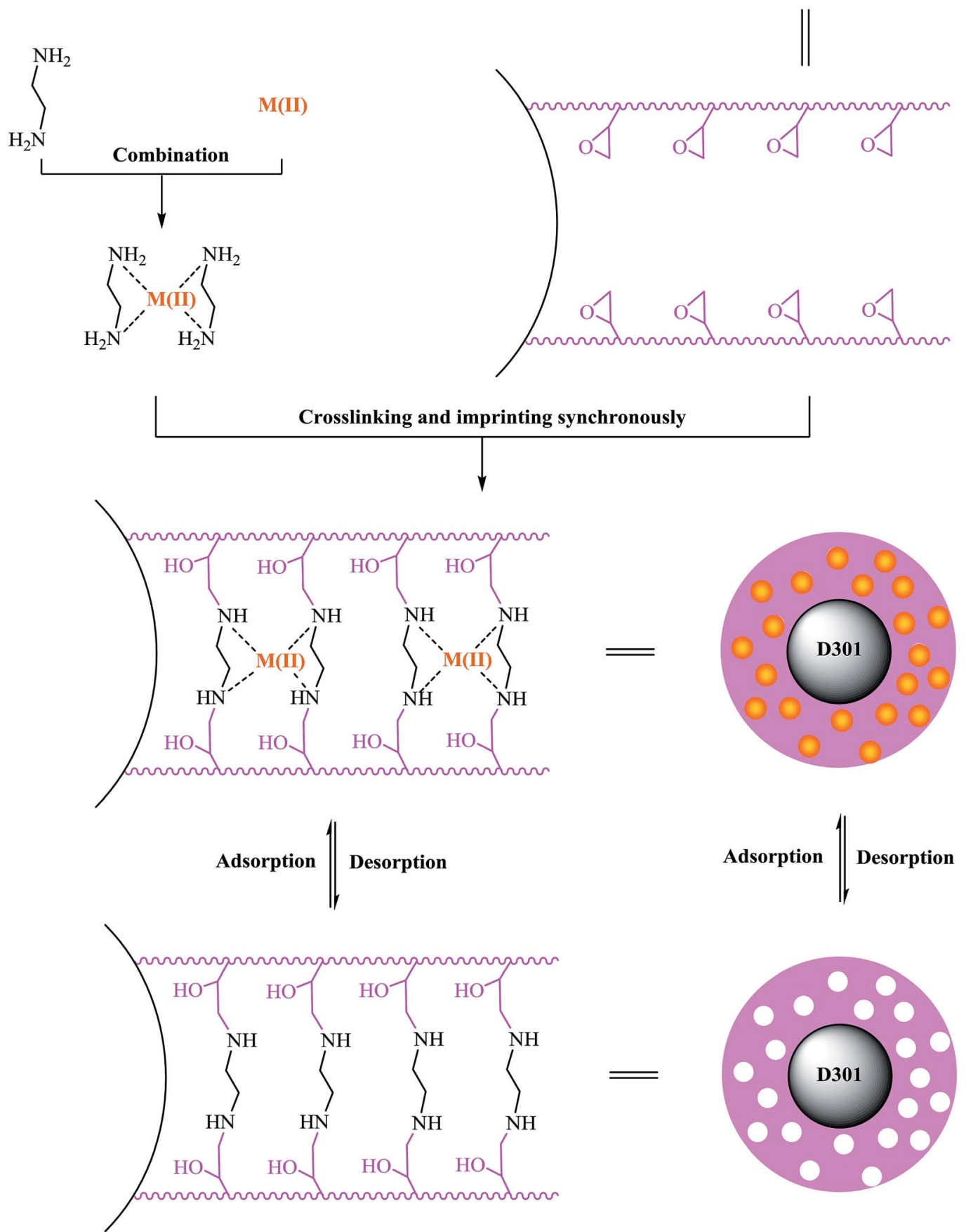

Fig. 2 The preparation process of metal ion-SIPs.

$200 \mathrm{ml}$ of mixed solution. The concentration of every metal ion was $1 \mathrm{mmol} \mathrm{\textrm {L } ^ { - 1 }}$ in the $\mathrm{Cd}$ (II) $/ \mathrm{Pb}$ (II)/Cu(II)/ $/ \mathrm{Zn}$ (II) multicomponent mixture. After adsorption reached equilibrium, the concentrations of all metal ions in the remaining solutions were determined using
ICP-AES. Distribution coefficients $\left(K_{\mathrm{d}}\right)$ and selectivity coefficients $(k)$ were calculated by eqn (2) and (3), respectively.

$$
K_{\mathrm{d}}=Q_{\mathrm{e}} / C_{\mathrm{e}}
$$




$$
k=K_{\mathrm{d}}\left(\text { template) } / K_{\mathrm{d}}\right. \text { (competitive ion) }
$$

\subsection{Repeated use experiment}

Reusability is an important scheduling criterion for an absorbent with a good application value. It also reflects the mechanical stability and chemical stability of the material. A desorption experiment was also carried out by a batch experiment using $1 \mathrm{~mol} \mathrm{~L}^{-1}$ of nitric acid as eluent. $1 \mathrm{~g}$ of exhausted adsorbent was placed in $10 \mathrm{ml}$ of eluent and stirred continuously at $298 \mathrm{~K}$ for $1 \mathrm{~h}$. In order to test the reusability, the adsorption-desorption procedure was repeated 10 times using the same SIPs.

\section{Results and discussion}

\subsection{Characterizations}

The SEM photographs of D301 and Cd-SIP are shown in Fig. 3S $\dagger$ and the particle distribution curves of D301 and Cd-SIP are shown in Fig. $4 \mathrm{~S} . \dagger$ It can be seen that the D301 still maintains good sphericity and surface morphology after the grafting and imprinting process.

The nitrogen adsorption-desorption isotherm is measured with a surface area analyzer by nitrogen absorption at $77 \mathrm{~K}$ using the Brunauer-Emmett-Teller (BET) method. Before measurements, the sample is degassed at $453 \mathrm{~K}$ in a vacuum for more than $3 \mathrm{~h}$. The surface area is calculated by the BET method from the adsorption branch in the relative pressure range $\left(p / p_{0}\right)$ of 0.005 to 0.1 . The surface properties including specific surface area $\left(S_{\mathrm{BET}}\right)$, total pore volume $\left(V_{\text {total }}\right)$ and average pore diameter (d) of different resins are listed in Table 1.

It can be seen that the macroporous D301 resin has large apertures. These macro-pores enable the grafting and imprinting process to be carried out efficiently. The decrease in $S_{\mathrm{BET}}, V_{\text {total }}$, and pore diameter could attest to the success of the grafting and imprinting process.

The FTIR spectra of different resins are shown in Fig. 3.

The infrared spectrum of D301- $g$-PGMA is similar to ref. 23 , and this attests to the form of D301-g-PGMA. It can be seen from the infrared spectrum of Cd-SIP that the characteristic absorption of epoxide rings $\left(1115 \mathrm{~cm}^{-1}\right)$ is greatly weakened and the characteristic absorption of the $\mathrm{C}-\mathrm{N}$ bond $\left(1260 \mathrm{~cm}^{-1}\right)$ is enhanced. Additionally, the Cd-SIP possesses high $\mathrm{N}$-content. These facts indicate that the ring-opening reaction of ethylenediamine and PGMA was carried out; that is, the grafted PGMA macromolecule chains were crosslinked by ethylenediamine. The crosslinking of PGMA

Table 1 The pore structure parameters of D301 and Cd-SIP

\begin{tabular}{llll}
\hline Sample & $S_{\text {BET }}\left(\mathrm{m}^{2} \mathrm{~g}^{-1}\right)$ & $V_{\text {total }}\left(\mathrm{cm}^{3} \mathrm{~g}^{-1}\right)$ & $d(\mathrm{~nm})$ \\
\hline D301 & 202.3 & 0.059 & 121 \\
D301- $g$-PGMA & 197.6 & 0.052 & 113 \\
Cd-SIP & 192.2 & 0.049 & 110
\end{tabular}

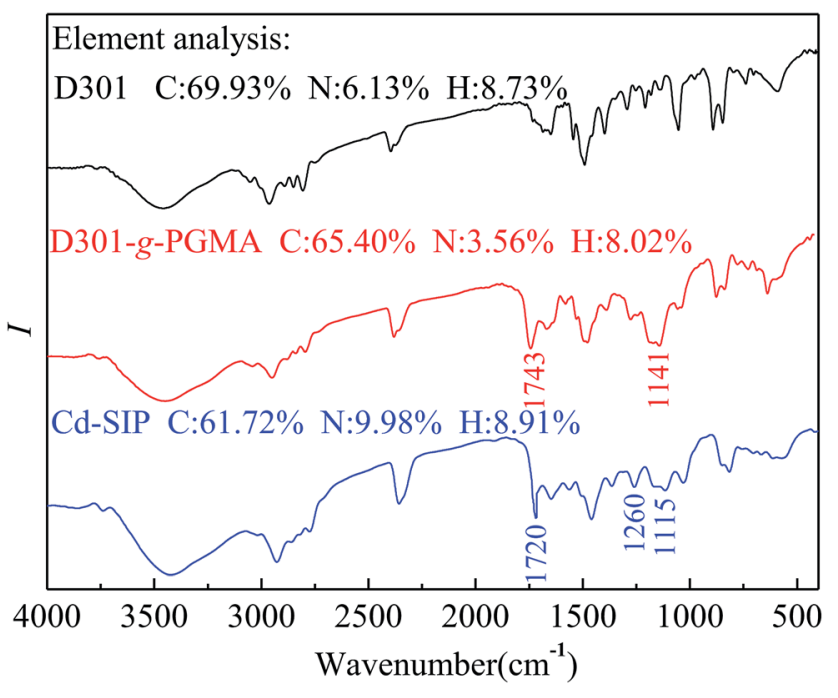

Fig. 3 FTIR spectra of D301, D301-g-PGMA and Cd-SIP.

results in the formation of imprinted cavities. By virtue of the specific effect of these imprinted cavities on the template, the Cd-SIP could selectively and efficiently adsorb the template.

\subsection{Kinetics adsorption curve}

The kinetics adsorption curves of SIPs and NIP towards different metal ions at $303 \mathrm{~K}$ are shown in Fig. 4.

It can be seen that the SIPs possess very strong adsorption ability and specific identification selectivity towards the template even in a binary solution. The saturated adsorption capacities of Cd-SIP towards Cd(II) in single and binary solutions are 2.23 and $2.21 \mathrm{mmol} \mathrm{g}^{-1}$ while the adsorption capacities of $\mathrm{Cd}$-SIP towards $\mathrm{Pb}$ (II) and of NIP towards Cd(II) are very low. The other three SIPs follow similar rules. The high adsorption ability and specific identification selectivity can be attributed to there being lots of imprinted cavities in the polymer thin layer. The selectivity is discussed below.

The Lagergren-first-order model (eqn (4) $)^{24}$ and pseudosecond-order model (eqn (5)) $)^{25}$ are usually used to test kinetics experimental data and describe the adsorption behavior of adsorbate onto adsorbent. The kinetics data are fitted with these two equations, and all parameters including the rate constant and correlation coefficient $\left(R^{2}\right)$ are summarized in Table 2.

$$
\begin{gathered}
\ln \left(Q_{\mathrm{e}, \mathrm{cal}}-Q_{t}\right)=\ln Q_{\mathrm{e}, \mathrm{cal}}-k_{1} t \\
t / Q_{t}=1 /\left(k_{2} Q_{\mathrm{e}, \mathrm{cal}}{ }^{2}\right)+t / Q_{\mathrm{e}, \mathrm{cal}}
\end{gathered}
$$

where $Q_{t}\left(\mathrm{mmol} \mathrm{g}^{-1}\right)$ is the adsorption capacity at time $t(\mathrm{~min})$, $Q_{\mathrm{e}, \mathrm{cal}}\left(\mathrm{mmol} \mathrm{g}^{-1}\right)$ is the calculated equilibrium adsorption capacity, and $k_{1}\left(\min ^{-1}\right)$ and $k_{2}\left(\mathrm{~g} \mathrm{mg}^{-1} \mathrm{~min}^{-1}\right)$ are the adsorption rate constants.

Compared with the $R^{2}$ value obtained from the pseudo-secondorder model, the $R^{2}$ value from the Lagergren-first-order model is closer to 1.0, and this reveals that the adsorption of Cd-SIP towards metal ions follows a Lagergren-first-order kinetics model. 

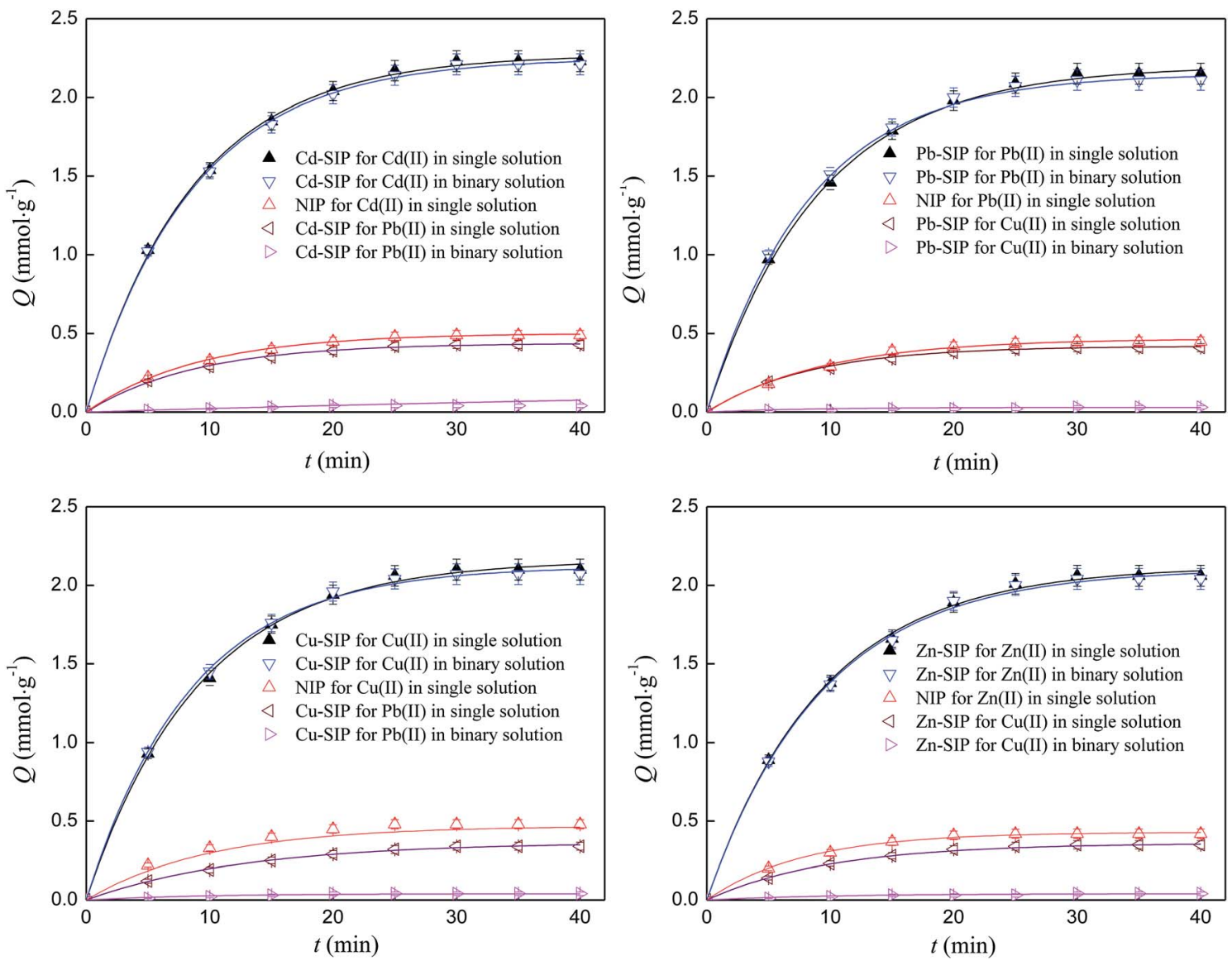

Fig. 4 Kinetics adsorption curves of SIPs and NIP. Initial concentration: $1 \mathrm{mmol} \mathrm{L}{ }^{-1}$; adsorbent dosage: $0.05 \mathrm{~g}$; volume of solution: $200 \mathrm{ml}$; temperature: $303 \mathrm{~K}$; pH: 6; number of repetitions: 3 .

Furthermore, the calculated equilibrium adsorption capacity $\left(Q_{\mathrm{e}, \mathrm{cal}}\right)$ of the Lagergren-first-order model is close to the experimental data, and this again indicates that the Lagergren-first-order model is more suitable for describing the adsorption process of Cd-SIP towards metal ions. The reason may be that the speed of combination of imprinted cavities with the template is fast and depends on the concentration of metal ions or the number of imprinted cavities.

The Elovich equation is used to confirm the chemisorption mechanism. ${ }^{26}$

$$
Q_{t}=\frac{1}{\beta} \ln (\alpha \beta)+\frac{1}{\beta} \ln t
$$

where $\alpha\left(\mathrm{mmol} \mathrm{g}^{-1} \mathrm{~min}^{-1}\right)$ is the initial adsorption rate, $\beta$ ( $\mathrm{g}$ $\mathrm{mmol}^{-1}$ ) is the desorption constant related to the extent of surface coverage and activation energy for chemisorption. The parameters are also summarized in Table 2.

It can be seen that the value of $R^{2}$ is close to 1 , and this indicates that chemisorption is the dominant interaction and

Table 2 The linear regression data of different models

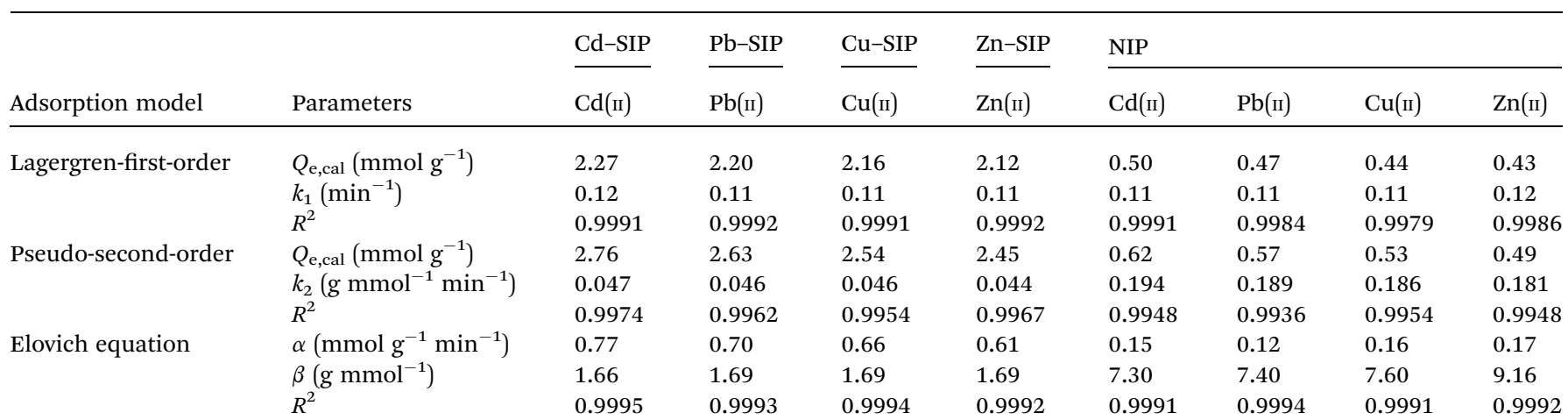






Fig. 5 Adsorption isotherm of Cd-SIP towards Cd(॥). Adsorbent dosage: $0.05 \mathrm{~g}$; volume of solution: $200 \mathrm{ml}$; temperature: $303 \mathrm{~K}$; adsorption time: $40 \mathrm{~min}$; $\mathrm{pH}$ : 6; number of repetitions: 3 .

the adsorption force is mainly the specific coordinated interaction of imprinted cavities towards the template.

\subsection{Adsorption isotherm}

The isothermal adsorption experiment is carried out under different metal ion concentrations and the adsorption isotherm of Cd-SIP towards Cd(II) is shown in Fig. 5. In order to obtain the adsorption behavior of $\mathrm{Cd}$ (II) on $\mathrm{Cd}-\mathrm{SIP}$, the adsorption isotherm is fitted using different isotherm equations which can describe the relationship between the equilibrium adsorption capacity and equilibrium concentration. The classical isotherm equations include the Langmuir (eqn (7)), Freundlich (eqn (8)), and Sips (eqn (9)) equations. ${ }^{27,28}$ The fitting curves are also shown in Fig. 5 and the parameters are shown in Table 3.

$$
\begin{gathered}
Q_{\mathrm{e}}=Q_{0} K_{\mathrm{L}} C_{\mathrm{e}} /\left(1+K_{\mathrm{L}} C_{\mathrm{e}}\right) \\
Q_{\mathrm{e}}=K_{\mathrm{F}} C_{\mathrm{e}}^{1 / n} \\
Q_{\mathrm{e}}=Q_{0} K_{\mathrm{S}} C_{\mathrm{e}}^{m} /\left(1+K_{\mathrm{S}} C_{\mathrm{e}}^{m}\right)
\end{gathered}
$$

where $Q_{\mathrm{e}}\left(\mathrm{mmol} \mathrm{g}^{-1}\right)$ is the equilibrium adsorption capacity, $Q_{0}$ $\left(\mathrm{mmol} \mathrm{g}^{-1}\right)$ is the monolayer adsorption capacity, $C_{\mathrm{e}}\left(\mathrm{mmol} \mathrm{L}^{-1}\right)$ is the equilibrium concentration, $K_{\mathrm{L}}\left(\mathrm{L} \mathrm{mmol}^{-1}\right)$ is the Langmuir adsorption equilibrium constant, $K_{\mathrm{F}}$ is the Freundlich adsorption equilibrium constant, $n$ is the heterogeneity factor, $K_{\mathrm{S}}$ is the Sips adsorption equilibrium constant, and $m$ is the dissociation parameter. The Sips equation could be reduced to the Freundlich equation when $K_{\mathrm{S}}$ approaches 0 or to the Langmuir equation when $m$ is close to 1 .

It can be seen that the $R^{2}$ of the Langmuir and Sips models are closer to 1 than that of the Freundlich model, and the value of $m$ in the Sips equation is also close to 1 . These indicate that the Langmuir and Sips equations could satisfactorily describe the isothermal adsorption experimental data, and the adsorption of Cd-SIP towards $\mathrm{Cd}$ (II) is a Langmuir-monolayer adsorption. This conclusion is consistent with the analysis of
Table 3 The fitting parameters and correlation coefficients of different equations

\begin{tabular}{lll}
\hline Model & Parameter & $\mathrm{Cd}(\mathrm{II})$ \\
\hline \multirow{2}{*}{ Langmuir } & $Q_{0}$ & 6.43 \\
& $K_{\mathrm{L}}$ & 1.17 \\
& $R^{2}$ & 0.9993 \\
Freundlich & $K_{\mathrm{F}}$ & 3.34 \\
& $n$ & 2.72 \\
Sips & $R^{2}$ & 0.9842 \\
& $Q_{0}$ & 6.15 \\
& $K_{\mathrm{S}}$ & 1.31 \\
& $m$ & 1.09 \\
& $R^{2}$ & 0.9994 \\
\hline
\end{tabular}

the Elovich equation that chemisorption is the dominant interaction because chemisorption must be monolayer adsorption. In the adsorption process, one $\mathrm{Cd}(\mathrm{II})$ imprinted cavity can only accommodate one template ion, so the adsorption must be monolayer adsorption.

\subsection{The influence of $\mathbf{p H}$ on adsorption capacity}

In general, $\mathrm{pH}$ has a great influence on the adsorption performance of $\mathrm{N}$-containing adsorbents towards heavy metal ions by affecting the protonation degree of the $\mathrm{N}$-containing groups. Therefore, the optimal $\mathrm{pH}$ needs to be determined experimentally in order to obtain the maximum adsorption performance. In this study, the adsorption experiment is performed at a $\mathrm{pH}$ below 7 in order to avoid the hydrolysis of heavy metal ions. The adsorption capacities of Cd-SIP, Pb-SIP, Cu(II)-SIP, and Zn(II)SIP towards the corresponding templates at different $\mathrm{pH}$ values are shown in Fig. 6.

It is obvious that all SIPs show a pH-dependent adsorption behavior. The adsorption capacity increases sharply when the $\mathrm{pH}$ changes from 0 to 4.0 and slowly reaches a maximum value at a $\mathrm{pH}$ of 6 . This phenomenon is similar to other $\mathrm{N}$ -

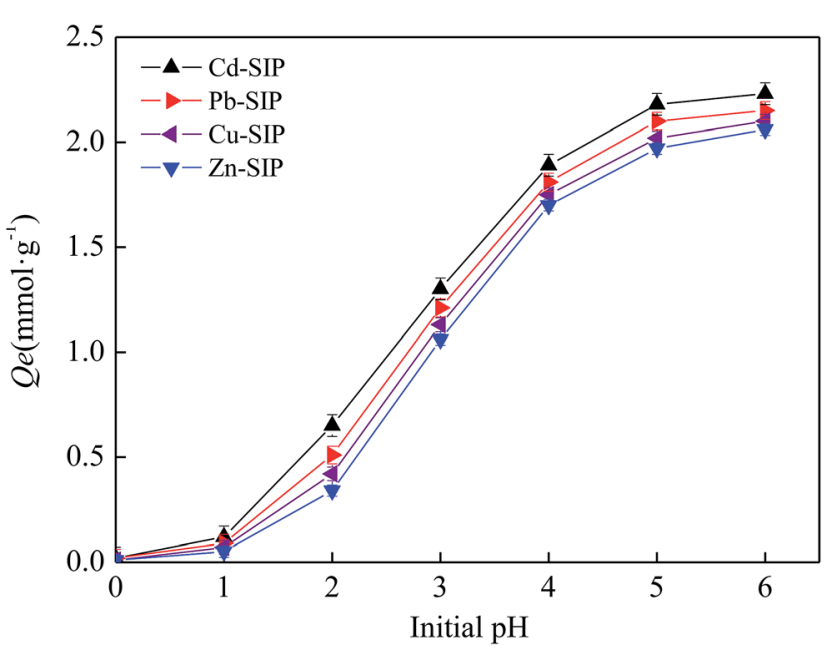

Fig. 6 The influence of $\mathrm{pH}$ on the adsorption capacity. Initial concentration: $1 \mathrm{mmol} \mathrm{L}^{-1}$; adsorbent dosage: $0.05 \mathrm{~g}$; volume of solution: $200 \mathrm{ml}$; temperature: $303 \mathrm{~K}$; adsorption time: $40 \mathrm{~min}$; number of repetitions: 3 . 
Table 4 Distribution coefficient and selectivity coefficient data



functionalized adsorbents. ${ }^{29-32}$ At a lower $\mathrm{pH}$, the majority (but not all) of the nitrogen atoms are protonated, so the active sites of the adsorbent are less available for metal ions due to the Donnan membrane exclusion from protonated amino groups of metal cations. Meanwhile, excessive protons also compete with metal ions which leads to a further decrease in adsorption performance. With the increase in the solution $\mathrm{pH}$, the protonation degree of the $\mathrm{N}$ atoms is lowered, which enhances the coordination interaction of amino groups with metal cations. The metal ions can be more easily bound on the resin active centers and the adsorption capacity increases with the increase in solution $\mathrm{pH}$.

Additionally, the low adsorption capacity at a $\mathrm{pH}$ of 0 indicates that the exhausted adsorbent can be regenerated using a strong acid as eluent.

\subsection{Adsorption selectivity}

Competitive adsorptions of different surface imprinted materials (Cd-SIP, Pb-SIP, Cu(II)-SIP, and Zn(II)-SIP) towards Cd(II)/ $\mathrm{Pb}$ (II)/Cu(II)/Zn(II) multicomponent mixtures are carried out by the batch method to research recognition selectivity. Table 4 summarizes the data of the distribution coefficients $K_{\mathrm{d}}$ and selectivity coefficients $k$.
It can be seen that the prepared surface imprinted materials have excellent selectivity towards the template. This is attributed to the imprinted cavities being complementary to the templates in size, shape and spatial arrangement of functional groups. The surface imprinted materials could effectively and selectively adsorb templates by virtue of these imprinted cavities with specific identification selectivity towards the templates.

The recognition selectivity, adsorption ability and regenerative property of the as-prepared SIPs in this study are higher than those of other IIPs prepared by other imprinted methods, and the comparisons of selectivity coefficient $(k)$, adsorption capacity and reuse times are listed in Table 5 .

It can be seen that the recognition selectivity, adsorption ability and regenerative property of SIPs are higher than those of other IIPs. This fully reflects the advances in the surface imprinting technology designed in this study. The designed surface imprinting technology in this paper can give the as-prepared SIPs more imprinting cavities, and more imprinting cavities predicts better recognition selectivity and adsorption ability.

\subsection{Desorption and reusability}

The desorption ability and regeneration of the exhausted SIPs must be considered because these are very important to

Table 5 The recognition selectivity comparison of different ionic imprinted materials ${ }^{a}$

\begin{tabular}{|c|c|c|c|c|c|c|}
\hline Imprinted method & Template & $\begin{array}{l}\text { Competitive } \\
\text { ion }\end{array}$ & $k$ & $\begin{array}{l}\text { Adsorption capacity } \\
\left(\mathrm{mmol} \mathrm{g}^{-1}\right)\end{array}$ & $\begin{array}{l}\text { Regenerative property } \\
\text { (Reused times) }\end{array}$ & Reference \\
\hline A & $\mathrm{Cu}(\mathrm{II})$ & $\mathrm{Zn}(\mathrm{II})$ & 3.29 & 0.92 & 4 & 8 \\
\hline $\mathrm{B}$ & $\mathrm{Cd}(\mathrm{II})$ & $\mathrm{Cu}(\mathrm{II})$ & 3.82 & 0.95 & - & 32 \\
\hline $\mathrm{B}$ & $\mathrm{Cd}(\mathrm{II})$ & $\mathrm{Zn}(\mathrm{II})$ & 1.15 & 0.95 & - & 32 \\
\hline $\mathrm{C}$ & $\mathrm{Pb}(\mathrm{II})$ & $\mathrm{Cd}(\mathrm{II})$ & 14.4 & 0.23 & 5 & 33 \\
\hline $\mathrm{C}$ & $\mathrm{Cd}(\mathrm{II})$ & $\mathrm{Cu}(\mathrm{II})$ & 0.9 & 0.71 & - & 35 \\
\hline $\mathrm{C}$ & $\mathrm{Cd}(\mathrm{II})$ & $\mathrm{Pb}(\mathrm{II})$ & 1.2 & 0.71 & - & 35 \\
\hline $\mathrm{C}$ & $\mathrm{Cd}(\mathrm{II})$ & $\mathrm{Zn}(\mathrm{II})$ & 1.4 & 0.71 & - & 35 \\
\hline $\mathrm{D}$ & $\mathrm{Pb}(\mathrm{II})$ & $\mathrm{Zn}(\mathrm{II})$ & 9.2 & 1.45 & - & 36 \\
\hline $\mathrm{E}$ & $\mathrm{Cd}(\mathrm{II})$ & $\mathrm{Cu}(\mathrm{II})$ & 81.3 & 2.23 & 10 & This study \\
\hline $\mathrm{E}$ & $\mathrm{Cu}(\mathrm{II})$ & $\mathrm{Cd}(\mathrm{II})$ & 59.9 & 2.10 & 10 & This study \\
\hline
\end{tabular}

${ }^{a} \mathrm{~A}$ is the bulk polymerization method; B is the emulsion polymerization method; $\mathrm{C}$ is the "grafting polymerization synchronized with crosslinking/ imprinting" method; D is the "pre-grafting polymerization and post-crosslinking/imprinting" method; E is the novel surface imprinted technique designed in this study. 


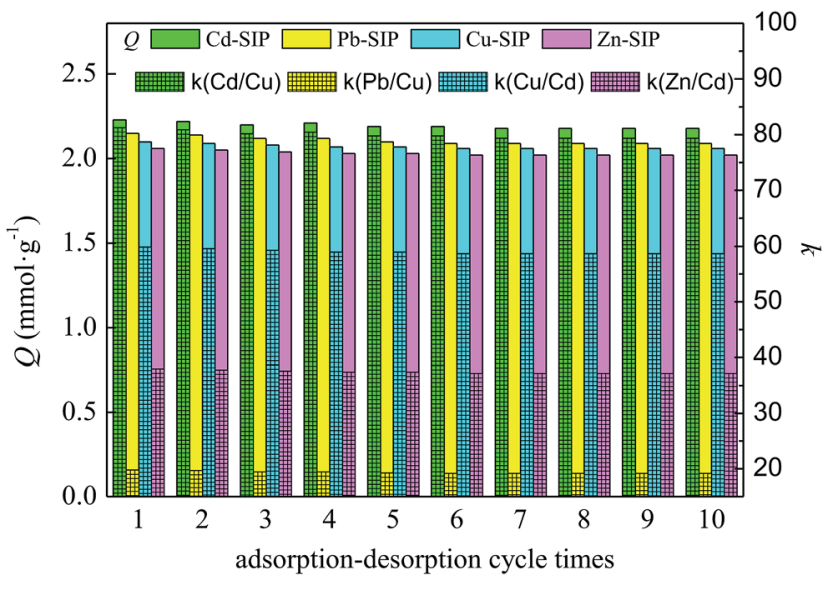

Fig. 7 Adsorption-desorption cycle of IIPs.

whether the adsorbent has a practical application value. The exhausted SIPs can be regenerated using $1 \mathrm{~mol} \mathrm{~L}^{-1}$ of nitric acid as eluent according to Section 3.4. In order to test the reusability of SIPs, the adsorption-desorption procedure is continuously repeated 10 times, and the results are shown in Fig. 7.

The results clearly show that the exhausted SIPs could be effectively regenerated by $1 \mathrm{~mol} \mathrm{~L}^{-1}$ of nitric acid aqueous solution, and the regenerated SIPs could be reused at least 10 times without a significant reduction in adsorption capacity or selectivity coefficient. This indicates that the stronger acid does not destroy the structure and functional groups of the imprinted cavities in the regeneration process, and the SIPs have good chemical stability and reusability. Overall, the as-prepared SIPs in this study are an effective adsorbent for selectively recognizing and adsorbing template metal ions.

\section{Conclusions}

A series of surface imprinted polymers (SIPs) using metal ions as a template are synthesized based on well-designed surface imprinting technology. The SIPs possess strong adsorption ability and excellent recognition selectivity towards the template, and the selectivity coefficients are higher than for other existing IIPs. The adsorption process could be well described by the Lagergren-first-order model and Langmuirmonolayer chemical adsorption. The SIPs have good chemical stability and reusability. The exhausted SIPs could be effectively regenerated and reused without significant reduction in adsorption capacity or selectivity coefficient.

\section{Conflicts of interest}

There are no conflicts to declare.

\section{Acknowledgements}

The authors gratefully acknowledge financial support for this work from the National Natural Science Foundation of China (21676258), the International Science \& Technology
Cooperation Program of China (2011DFA51980), the Natural Science Foundation of Shanxi Province (201601D102021, 2015021045), the Key Research and Development (R\&D) Projects of Shanxi Province (201803D31061, 2015081043), and the International Scientific and Technological Cooperation Projects of Shanxi Province (201803D421080).

\section{Notes and references}

1 L. M. Madikizela, N. T. Tavengwa, H. Tutu and L. Chimuka, Trends Environ. Anal. Chem., 2018, 17, 14.

2 J. Wackerlig and R. Schirhagl, Anal. Chem., 2016, 88, 250.

3 L. M. Madikizela, N. T. Tavengwa and L. Chimuka, J. Pharm. Biomed. Anal., 2018, 147, 624.

4 S. Boulanouar, S. Mezzache, A. Combès and V. Pichon, Talanta, 2018, 176, 465.

5 R. Gui, H. Jin, H. Guo and Z. Wang, Biosens. Bioelectron., 2018, 100, 56.

6 P. Luliński, Mater. Sci. Eng., C, 2017, 76, 1344.

7 Z. Zhou, D. Kong, H. Zhu, N. Wang, Z. Wang, Q. Wang, W. Liu, Q. Li, W. Zhang and Z. Ren, J. Hazard. Mater., 2018, 341, 355.

8 H. Liu, D. Kong, W. Sun, Q. Li, Z. Zhou and Z. Ren, Chem. Eng. J., 2016, 303, 348.

9 H. Guo, Y. Liu, W. Ma, L. Yan, K. Li and S. Lin, J. Hazard. Mater., 2018, 348, 29.

10 D. Li, T. Tu, M. Yang and C. Xu, Talanta, 2018, 184, 316.

11 Z. Zhang, D. Niu, Y. Li and J. Shi, Appl. Surf. Sci., 2018, 435, 178.

12 G. Zhu, X. Gao, X. Wang, J. Wang and J. Fan, J. Chromatogr. A, 2018, 1532, 40.

13 K. Eersels, P. Lieberzeit and P. Wagner, ACS Sens., 2016, 1, 1171.

14 H. Liu, T. Ni, L. Mu, D. Zhang, J. Wang, S. Wang and B. Sun, Sens. Actuators, B, 2018, 256, 1038.

15 J. He, H. Shang, X. Zhang and X. Sun, Appl. Surf. Sci., 2018, 428, 110.

16 X. Wang, P. Huang, X. Ma, X. Du and X. Lu, J. Chromatogr. A, 2018, 1537, 35.

17 H. Gong, S. Hajizadeh, L. Jiang, H. Ma and L. Ye, J. Colloid Interface Sci., 2018, 509, 463.

18 Y. Xiong, Y. Song, Q. Tong, P. Zhang, Y. Wang, Z. Lou, F. Zhang and W. Shan, Sep. Purif. Technol., 2017, 177, 142.

19 C. Philip and K. S. Devaky, Mol. Catal., 2017, 436, 276.

20 B. Gao, H. Liu and K. Cui, Sens. Actuators, B, 2018, 254, 1048. 21 B. Gao, L. Zhang and Y. Li, Mater. Sci. Eng., C, 2016, 66, 259. 22 B. Gao, J. Meng, Y. Xu and Y. Zhang, J. Ind. Eng. Chem., 2015, 24, 351.

23 F. Q. An, M. Li, R. Y. Wu, T. P. Hu, J. F. Gao and Z. G. Yuan, Hydrometallurgy, 2017, 169, 356.

24 S. Lagergren and K. Svenska, Vetensk. Akad. Handl., 1898, 24, 1.

25 Y. S. Ho and G. McKay, Process Biochem., 1999, 34, 451.

26 S. H. Chien and W. R. Clayton, Soil Sci. Soc. Am. J., 1980, 44, 265.

27 I. Langmuir, J. Am. Chem. Soc., 1916, 38, 2221.

28 H. M. F. Freundlich, Z. Physiol. Chem., 1906, 57, 385. 
29 H. Z. Cui, Y. L. Li, S. Liu, J. F. Zhang, Q. Zhou, R. Zhong, M. L. Yang and X. F. Hou, Microporous Mesoporous Mater., 2017, 241, 165.

30 C. R. T. Tarley, M. Z. Corazza, F. M. Oliveira, B. F. Somera, C. C. Nascentes and M. G. Segatelli, Microchem. J., 2017, 131, 57.

31 F. Q. An, Y. Wang, X. Y. Xue, T. P. Hu, J. F. Gao and B. J. Gao, Chem. Eng. Res. Des., 2018, 130, 78.
32 F. Zhu, L. Li and J. Xing, J. Hazard. Mater., 2017, 321, 103. 33 S. Mishra and N. Verma, Chem. Eng. J., 2017, 313, 1142. 34 Y. Liu, X. Hu, Z. Liu, M. Meng, J. Pan, Y. Jiang, L. Ni and W. Wu, Chem. Eng. J., 2017, 328, 11.

35 K. Huang, Y. Chen, F. Zhou, X. Zhao, J. Liu, S. Mei, Y. Zhou and T. Jing, J. Hazard. Mater., 2017, 333, 137.

36 R. Msaadi, S. Ammar, M. M. Chehimi and Y. Yagci, Eur. Polym. J., 2017, 89, 367. 